\title{
EL HOSPITAL "SAN VICENTE DE PAUL", DE CUENCA
}

\author{
Dr. GRAULIO POZO \\ Directur del Hospital
}

\section{Sintesis histúrica:}

A pesur de que cuatudo al Virrey del Per'u, Marqués de Cuñele, ordenó a Gil Ramirez Dávalus la funclación de Cuenea en 1557, con la ubligarión de sen̂alar un sitio para hospital de esparoles e irulios, no se construyó éste, simo casi dos siglos después, o sea en 1740 máx o menos. El tieal que ha estade ubicado en la escuuina de lo gle ahora son täles Crian Colombia y Lils Cordero, fue cedido por la Real Audiencia $y$ el Obispo de Quito a la Orden Bethicmita. Posterinimente, par orden del Rey, se functó el Convento de Bethlemitas junto al IIospital, con la consigna de que los Bethlejnitas cuiden de la asistoncia y curación de Jos enfermos, sogín C'édula Real que tue ejeculada en 1783, en tiempo clel Goberaador Dua doseph Vallejo.

Fray Santiago de las A nimas fue el Dircetor de este Hospita]. Como pasados algumos años, el Hospital resultarn extrecho, se buscó un lugar mits apropíado y, a modiaclos del siglo pasado, se trasladó a San Blas, al sur del á:tual Pirque Flurtưlo cle Mendoza. Altí tampoco se pudo dar la cumadiclad que requería tal servicio y hubo necsesirlad de clausurarlo casi abtes de ensayarlo en forjua efectiva. En este Jirspilal de Sàn BIas, paru ruya instalación y fumcionamiento cuntelbuyó activanenle el Sr. Dr, Francisco Cuesia, son sus valiosos servicius prof tsionales.

Por el ar̆o 1868, el Presidente de li República, IJr. IJn, Gabricl García Mo. retro, ws su visita a Cucouca, orifenó findar el actual Hospital "Sun Vicende de Paúl", a la orilla sur del río Matam dero.

Para lievar a feliz términu su deseo, contrató en Europa a las. Hemanas de la Caridad po: sen las I]amadas, por vocación, al cuidado de los enfermos.

Hizo grandes asignectanes de dinor para llovar a cubo la construcción $y$ t'usındo ol edificio estuvo en estado dta prestar servicios, el 28 de diciembre de 1872, se inauguraba solemnemente el nuevo Hospital, La administración se confió a la Conferencia de San Vicente de I’aúl, siendo los principales dirigente los Dres. Marsanc Cueva y Josts Trancisco Moscoso y el Sr. Marianze Estrella.

Posterismente se ha ido ampliands con la consterucción de una seric de edi- 
ficius, subvencionedos lis mayor parte wet las Juntas de Asistencia Social y en algunes ocasionos por filántropos como los Sres. Cornelio Merchán y Miguel Delgado. En 1945, al Sorvicio Cooperativo Interamerjt:ano colaburó para la reconstutcejón de una parte del HosHital.

E] Hospita[ "San Vicente de Paúf" cuenta eu la actualidad con los siguientes servicios:

\section{Servicios de elínica:}

Mujeres: 3 salas won 54 camers Hombris: 2 salas con 40 camas Tutal de tamas: 94.

\section{Servieivs de cirugia:}

Mujeres: 1 sijat eon 19 camas

Hombres: 1 sala con 20 ramas

Total de camas: 39 .

Servicio de pediatría:

1 salu con 26 camas.

Servicio de ginecología:

1 sala con 16 camas.

\section{Servicio de obstetriciu:}

1 sala con 16 camas.

\section{Servicio de ponsionado:}

Hombres: 6 cames

IMujeres: 8 camas.

\section{Servicio de semipensionado:}

Honibres: $\mathbf{6}$ eamas

Mujeres: 8 camas.

Servicio de infectocontagiosos:

Hombres: 22 camas

Mujeres: 25 camas

Total de camas: 264.

\section{Servicio de consulta externa:}

\begin{tabular}{|c|c|c|}
\hline \multicolumn{3}{|c|}{ Part casos clínjeus } \\
\hline 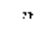 & $"$ & quirúrgicos \\
\hline " & $"$ & otorrinolaringológicus \\
\hline ": & $\Rightarrow$ & olta]mológicos \\
\hline " & $"$ & ginecológieos \\
\hline$\Rightarrow$ & $"$ & ortopédicus \\
\hline
\end{tabular}

Servicio de emergencia

Servicio de recuperación

Laboratorio clínico

Laboratorio de Anatomía Patológica Gabinetes para Electro-radiografia. Electrocardiografía y Metabulismo I3ustst.

Botica, Biblioteca, Ropería y Cucina. EI ingreso de parientes a Ios diferentes strvieios tiene un promedio de 4.500 al año (datos tomados desdo. 1958). Lis casos atendidos en lus distintos servicios do consulta cxterna leמau a] mümeju de 120 djarìss. En e] Gabinete de Electru-radiología se eontrolar un rúmearo no menor de 20 diarios.

El laboraturio c:inico ha realizado durarste el presente año, el número de 27.000 exámeries. Fin el laburatorio de Anatomia Patológica, cir 1963, se han practicado 1.300 exámenes histojato- 
lógicos de :naterial quirúrgico y 50 neeropsias. Durantc el presente año, hasta la fecha, se llevan hechos 2.000 exáme. nes histopatológicos y 65 necropsias.

El promedio mensual de intervenciones quirurgicas as de 85 , número que râ en aumento, subre lodo en to que respecta a casus de Ortopedia.

Para цли mejor organización del servieio hospitalario y por ser éste un hospital docente, se han sugerido algunas ìnovaciones, algunas de las cuales ya están llevíndose a lia prácticz: la formación de una Junta Directiva dol Hospifal, cun el lir de conseguir un co-go. biemo da la Asistencia Sucial y de la Fircultad te Medicina, en el maneju administrativo y técnico del Huspitáli

Organizdcion departanestal de lus diversos survicios de medicina interna, cirugía, pediatría, obstetricia y rínecojogía. Están orgenización estará ligada intimamente a la formación de las respectivis consultas externas do caráter netamente deparianental.

Cun el asesoratniento de los niembros del Proyecto HOPE so osián pirneandu los siguientes Cumités: el de Uisturitis Clínitas; el de Internos y Revidenles y el de Futadística.

Apremiades por la necesidad do unificar liss servicios de cirugía lantu por ruzones tócniens corne administrativas, se están rediłando al lia fncha modificacioncs en este rlcyatlamenty, con el fiv de entializar las suias de openacionos $y$ el servicio de esterilización. A fin die que el servicio de podiatría rinda el bejelicu que desumrs, se ha plabeactiv, y muy jucunto scrá rcalidład, el funcionanisento de un "Poliolínico In-
Pantil", aunque pequeño, pero con las comodidadiai necesarias para atender casos de medicina interna, cirugía, prematuros, lactantes y una sección de rehidratacion.

Asimistro, en vista de la necesidad udgente de contar cun ut departamenc) de rehabilitureión, se estí andecuanda un locitl para cste servicio, en el cual, Jos pacientes correspondientes a la espceialidad de ortopedia, tratados en al Huspital, no clueriarán abandonados, simo túc serán sometidos a tratamientos físico-terapéuticos, a fit1 de ponerlos en cotuliciones ayptas para el trabaja y desarroito de sus actividades normales.

Como el Proyecto HOPE nos ha brindadn la opnrtunidad de rcalizar un programe ampitio en nuestro Ifustjital, en el jróximo tress se iniciará un curso parą auxiliares de enfermería y cursoi para lécruicos de luboratorio bioquímico, laboratorio aratomi-patológiro y de rayos X. Adelnás se está obteniendr un asesoratrientu en servicios médicos y para-inćdicos.

Fl porsonal téchico $y$ adíninistrativo cun que cuente el Hospital "San Vicente de ["átil", es el siguiente:

1 Médico Director

1 Allininistrador

1 Sectetario-Jefe de Fstadistiua

1 Cuntaclur

$\perp$ Ayudinde de Conlahilidad y de Fsladística

1 Bibliotccaria - Auxiliar de Esqadística.

2 To!eforisistas

1 Inspector de obra $y$ comstrucciones. 
1 Médico Jefe dol gabinete de veletro-radiología

1 Médico jeto del laboratorio clínico

1 Médico jefe del laboratorío da analoní́a patológic:a

1 Médico ayudante del laboratorios clínico

1 Médico de consulta externa y encargartir del gabinetc de elcetrocardiología $\mathrm{y}$ molabolismo basal

2 Médicos de consultal externa, para cirugía y méjicina interna.

2 Médicos de consulta externa, pära oftalmología $y$ oldurrinoluringologín

2 Médicos residentes

1 Médivo jefe de anestesiología

1 Farmacéutico

3 Enfermerus

16 Hermanas de la Caridad, udgunas eon títulos do enformeras

18 Auxiliares de entermeria

2 Auxiliares de laboratorio clínico

2 Auxiliares del gabinete de electru-radiologia

1 Auxiliar del laboratorio de anatomia patsiogien
5 Alumnos internos egeresados, con sucldo $y$ ad-honorem

1 Ayudante de anestesia, egresado

3 Auxiliares de botica

2 Asistentes veludores

20 Asistentes de sorvicia

2 Porteros - 1 Mandadero y 12 personas para lavandería, cueina, costura.

Como el Hospìal "Sart Vicentc de Paúl', ya do eś solamente tit Hospital local, sino regiona], puesto que a esti casn de salud acuder enfermos de la tegión Oriental, del Azuay, Cuñar y aún de Loja y Él Oro, cada ve\% ek més pequıeño, razón por la que la II. Junta fle Asistervia Social del Aziay, Cañar y Morona Suntiago, con clara visión deI fututro, ostá interesada en llevar a cabo la construcción de un Hospital moderno y amplio, con lo que (reemrus que se subsanarán las grandes dificultades que ahora temernus para brindar un serviciv eficiente a todos los enfermos que necesitan ser hospitulizados. Lra cunstrucción de este nuevo Jespilal se iniciará seburamente a mediados deI próximo año. 